\title{
Diacronie
}

Studi di Storia Contemporanea

$\mathrm{N}^{\circ} 18,2 \mid 2014$

Le esposizioni: propaganda e costruzione identitaria

\section{Ahdaf Soueif, Il Cairo, la mia città, la nostra rivoluzione}

\section{Luca Zuccolo}

\section{(2) OpenEdition}

\section{Journals}

\section{Edizione digitale}

URL: http://journals.openedition.org/diacronie/1543

DOI: $10.4000 /$ diacronie. 1543

ISSN: 2038-0925

\section{Editore}

Association culturelle Diacronie

Notizia bibliografica digitale

Luca Zuccolo, «Ahdaf Soueif, II Cairo, la mia città, la nostra rivoluzione », Diacronie [Online], N 18, 2 I

2014, documento 17, Messo online il 01 juin 2014, consultato il 24 septembre 2020. URL : http:// journals.openedition.org/diacronie/1543; DOI : https://doi.org/10.4000/diacronie.1543 


\section{Diacronie}

N. 18 | 2|2014 Le esposizioni: propaganda e costruzione identitaria

17/

\section{RECENSIONE:}

\section{Ahdaf SOUEIF, Il Cairo, la mia città, la nostra rivoluzione, Roma, Donzelli Editore, 2013, 258 pp.}

a cura di Luca ZUCCOLO *

«Da un capo all'altro del paese, gli orizzonti delle persone comuni sono cambiati. La mappa del Cairo si è rinnovata; nei nomi dei luoghi risuonano energie e significati del tutto nuovi»1. Questo è il messaggio più profondo del libro di Ahdaf Soueif, in cui l'autrice - scrittrice, giornalista e commentatrice politica e culturale ${ }^{2}$ - ci ha voluto lasciare una descrizione accurata ed emozionante della rivoluzione che ha scosso e scuote ancora oggi l'Egitto.

Il saggio, in uno stile agile e coinvolgente attraversa più generi da quello memorialistico a quello biografico, passando attraverso il racconto e il saggio politico. Come scrive la stessa Soueif, rivolgendosi ai suoi lettori:

questo libro non è il diario di una vicenda conclusa; è il tentativo di farvi entrare, di rendervi partecipi di un evento che stiamo ancora vivendo. E scriverlo è problematico per due ragioni. Una è che, mentre i diciotto giorni sono blindati nel passato, la rivoluzione e lo sforzo per difenderla sono ancora in corso in uno scenario che cambia di giorno in giorno. La seconda ragione è che, anche se voi lettori vi trovate in un futuro che io non conosco, vorrei comunque consegnarvi una storia che vi aiuti a colmare la distanza tra il momento in cui si conclude questo racconto e la situazione in cui verserà l'Egitto nel momento in cui voi leggete.

\footnotetext{
${ }^{1}$ SOUEIF, Ahdaf, Il Cairo, la mia città, la nostra rivoluzione, Roma, Donzelli Editore, 2013, p. 174 (ed. ing. Cairo, My City, Our Revolution, London, Bloomsbury Publishing, 2012).

2 Autrice di diversi romanzi e saggi tra cui ricordiamo: SOUEIF, Ahdaf, In the Eye of the Sun, New York, Random House, 1992; Sandpiper, London, Bloomsbury, 1996; ID, The Map of Love, London, Bloomsbury, 1999; ID, Mezzaterra: Fragments from the Common Ground, New York, Anchor Books, 2004; Soueif è molto attiva anche nel campo politico - come testimonia il saggio qui recensito - e nel campo culturale come fondatrice del Palfest (Festival della letteratura palestinese) attivo dal 2008, < http://palfest.org/ > [consultato il 9 agosto 2013].
} 
Mentre scrivo, vi immagino tra qualche tempo, nelle prossime settimane, mesi, con questo libro in mano a scorrere le mie parole, e mi domando quale realtà avrete sotto gli occhi. Sarà la realtà che noi, oggi, mentre scrivo, stiamo costruendo con il nostro operato, con la lotta, con la morte.

Queste sono le ultime ore che io e il mio testo trascorriamo insieme; le ultime ore che ho a disposizione per scrivere per voi, a voi3.

Attraverso le pagine di Soueif, quindi, ascoltiamo la voce di un insider, che non solo descrive i fatti del Cairo e di Tahrir, ma li contestualizza nel più ampio quadro sociopolitico egiziano fornendoci uno spaccato della situazione del Paese, della caduta del regime di Mubarak e del difficile cammino verso una democrazia reale più partecipativa, che diventi espressione delle richieste della maīdan (piazza): "Pane, libertà e giustizia sociale”.

Il saggio si inserisce in un'ampia corrente saggistica che fin dai primi mesi post rivoluzionari ha espresso pareri e commenti sulla rivoluzione del Cairo 4 e sulle sue conseguenze, spesso relazionandola alle precedenti espressioni delle primavere arabe nordafricane. Il libro della Soueif, tuttavia, non è solo questo. L'autrice come si è visto poc'anzi cerca non solo di descrivere e commentare i fatti, ma di renderne partecipe il lettore attraverso il suo sguardo e le sue "memorie". Il saggio non ha il fine di convincere o di propagandare una visione particolare degli avvenimenti ma, al contrario, ha lo scopo di far conoscere e di rendere compartecipi i lettori dei fatti in modo che chiunque possa capire o per lo meno percepire le sofferenze e le motivazioni che hanno spinto un intero popolo a scendere in piazza e a manifestare pacificamente per riappropriarsi dei propri spazi urbani, sociali e dei propri diritti civili e umani.

La storia raccontata da Soueif, pertanto, non è solo la narrazione degli eventi di piazza Tahrir, ma è soprattutto l'espressione di come i cittadini si siano riappropriati della maīdan e con essa di un nuovo modo di fare politica. Questo libro è il racconto di come gli egiziani abbiano reinterpretato, usandoli in modo differente due diversi spazi sociali, la piazza, appunto, e i social network. Per la prima volta al Cairo, il ruolo dei social network come twitter e facebook si è dimostrato rilevante non solo a livello aggregativo ma proprio come spazio sociale alternativo in cui si poteva liberamente esprimere il proprio parere rivoluzionario e al contempo farsi ascoltare a livello

3 SOUEIF, Ahdaf, Il Cairo, la mia città, la nostra rivoluzione, cit., pp. 59-60.

4 Solo per citare alcuni titoli: AL-ASWANI, 'Ala, La rivoluzione egiziana, Milano, Feltrinelli, 2011; QUIRICO, Domenico, Primavera araba. Le rivoluzioni dall'altra parte del mare, Torino, Bollati Boringhieri, 2011. 
globale5. La forza che gli attivisti di piazza Tahrir hanno messo in campo - anche grazie ai social media - ha permesso loro di bypassare, nonostante l'accanita resistenza, i media tradizionali controllati dal regime e dall'esercito, un esempio che sarà seguito in tutte le successive manifestazioni come ha recentemente testimoniato Gezi Parkı a Istanbul.

Il racconto della Soueif, nondimeno, è anche l'espressione delle profonde divisioni che percorrono la società egiziana frazionata in molteplici gruppi e percorsa da cleavages religiosi e culturali molto rilevanti con cui piazza Tahrir si è dovuta confrontare e che non sempre è riuscita a superare con il suo spirito unitario e collettivo. Infine, questo saggio è un racconto biografico della stessa Soueif, la quale intervallando le sue esperienze a Tahrir con i suoi ricordi di infanzia e con la sua vita praticamente tutta la sua famiglia è coinvolta a vario titolo nella rivoluzione - ci propone uno spaccato socio-storico dell'Egitto che va molto più in profondità nella storia recente cairota-egiziana e ci permette di osservare alcune delle cause della rivoluzione e soprattutto i rapidi e notevoli mutamenti subiti dalla società cairota ed egiziana negli ultimi decenni ${ }^{6}$.

Questo diario si suddivide in quattro capitoli che alternano il racconto dei primi 18 giorni di rivoluzione del 2011, quelli che diedero avvio a tutto e portarono alla caduta di Mubarak, al commento dei risultati e delle conseguenze di quei diciotto giorni e, soprattutto, come la rivoluzione con i suoi protagonisti sia andata avanti nei due anni successivi (2011-2012) fino alle elezioni presidenziali che hanno visto la vittoria di Morsi e dei Fratelli Musulmani e nei mesi immediatamente seguenti.

Il primo capitolo, Diciotto giorni, propone una descrizione dell'inizio e delle prime fasi della rivoluzione di piazza Tahrir fino al giorno 1 febbraio 2011. Soueif racconta il suo iniziale coinvolgimento nelle manifestazioni di piazza e propone una prima genealogia dei principali personaggi che la circonderanno in quei diciotto giorni e nei mesi/anni successivi.

Il secondo capitolo, dal significativo titolo Interruzione, Otto mesi dopo: Ottobre 2011, lascia per un attimo la memoria di Tahrir e fa un salto in avanti per descrivere

\footnotetext{
5 L'ultima frase del libro di Soueif è significativa a questo riguardo: "poiché i sistemi che opprimono e sfruttano la gente sono diventati globali, globale è diventata la nostra resistenza». SOUEIF, Ahdaf, op. cit., p. 236.

6 Relativamente alla storia egiziana recente si veda: GOLDSCHMIDT, Arthur Jr., Modern Egypt: The Formation of a Nation State, Boulder, Colo., Westview Press, 2004; IBRAHIM, Saad Eddin, Egypt, Islam, and Democracy, Cairo, American University in Cairo Press, 1996; NASSER, Gamal Abdel, The Phylosophy of the Revolution, Cairo, Egyptian State Printing Office, 1955; SADAT, Anwar, In Search of Identity, New York, Harper \& Row, 1978; VATIKIOTIS, P. J., The History of Modern Egypt from Muhammad Ali to Mubarak, Baltimore, Johns Hopkins University Press, 1991.
} 
alcuni dei primi effetti della rivoluzione. È un capitolo di commento anche se l'aspetto memorialistico e biografico permangono preponderanti. Accanto alle constatazioni sui risultati ottenuti dalla maīdan, Soueif descrive alcuni fatti salienti avvenuti durante l'estate del 2011 e soprattutto i colpi di coda di un regime non ancora completamente abbattuto. Questi sono i mesi di dominio dell'esercito e dell'inizio della repressione più violenta delle manifestazioni. Sono i mesi in cui maggiormente si contano i martiri, i quali, anche dopo la loro morte, accompagneranno le manifestazioni, capeggeranno i cortei e faranno sentire la loro voce a piazza Tahrir attraverso il dolore dei loro familiari.

Il terzo capitolo, Diciotto giorni (seconda parte), descrive la rivoluzione dal 2 febbraio all'11 febbraio, quando la piazza otterrà la sua prima vera vittoria riuscendo a far cadere il presidente Mubarak. Il filo del racconto e delle memorie riprende da dove è stato interrotto nel primo capitolo e descrive l'escalation degli avvenimenti che hanno portato alla caduta del regime.

Infine, il quarto e ultimo capitolo Diciotto giorni non sono bastati racconta il difficile cammino post-rivoluzionario, il confronto con lo Scaf (Consiglio Supremo delle Forza Armate), la repressione da parte di esercito e polizia e la resistenza della maīdan di fronte ad ogni nuovo tentativo di far scemare le proteste. In questo capitolo, come nel secondo, accanto al racconto diaristico della rivoluzione e dei mutamenti sociali da essa apportati, Soueif descrive la peculiare situazione politica egiziana, la frammentazione dei partiti e l'inconsistenza del parlamento politicamente diviso a metà tra l'esercito e il vecchio regime. Vengono descritte le azioni politiche dei diversi gruppi coinvolti nella rivoluzione e di quelli che si sono mantenuti ai margini, si descrivono le perduranti divisioni socio-religiose e politiche, la mancanza di accordi e compromessi fino alle elezioni presidenziali del maggio 2012, che dopo il ballottaggio hanno visto la vittoria dei Fratelli Musulmani. La fine del capitolo e del saggio è lasciata alle domande sul futuro dell'Egitto e sull'operato di Morsi, il quale nonostante la sua apertura al dialogo durante le elezioni, non sembra veramente propenso a dialogare da quando è salito al potere, e tanto meno a soddisfare le richieste della piazza: "pane, libertà e giustizia sociale”.

Il testo è corredato da una prefazione in cui l'autrice spiega come sia giunta a scrivere questo diario, da un apparato di note molto utile per approfondire quanto descritto nel libro grazie a diversi rimandi a siti internet e contenuti multimediali. Attraverso questi è possibile osservare quanto succedeva e succede al Cairo e in Egitto. Infine, il saggio è completato da una breve storia dell'Egitto moderno, utile per acquisire le informazioni di base sulle tappe politiche cha hanno condotto alla 
rivoluzione di Tahrir, e da due brevi note della traduttrice Nicoletta Poo la quale fornisce una breve cronologia dei fatti salienti dei diciotto giorni che hanno cambiato l'Egitto e un'utile micro biografia di Ahdaf Soueif e della sua famiglia.

Diario, memoria, saggio, autobiografia, molti sono i generi utilizzati da Soueif per descrivere una parte della sua vita e dell'Egitto intero. Sicuramente il testo proposto è una visione personale e peculiare degli avvenimenti, ma rappresenta anche uno sguardo disincantato su queste vicende, a volte drammatiche, che attraverso l'esperienza dell'autrice consente a noi lettori occidentali di calarci all'interno della protesta di Tahrir, capirne le motivazioni, le divisioni, le sfaccettature e le contraddizioni. Quello che è certo, come dimostrerà in seguito anche la "primavera" turca a Gezi, è che quanto è accaduto e continua ad accadere a Tahrir, al Cairo e in tutto l'Egitto è un esempio di cambiamento e rinnovamento. Questa rivoluzione, infatti, non solo ha utilizzato mezzi e strumenti innovativi come internet, i social network e i video filmati; ha riscoperto anche la piazza come luogo di incontro-scontro e di condivisione di opinioni, come salotto politico alternativo e collettivo rispetto alle obsolete stanze del potere, chiuse in palazzi inaccessibili e poco rappresentative del popolo e dei suoi sentimenti. I fatti di Tahrir, come quelli successivi di Gezi, nondimeno, si sono presentati anche come l'espressione di un malessere generale che coinvolge primariamente la classe media, che è scesa in piazza non solo per difendere i propri e gli altrui diritti, ma anche per proporre un nuovo e alternativo modo di fare e di intendere la politica e l'economia. Il grido di Tahrir, "pane, libertà e giustizia sociale", non è solo una richiesta di maggiori diritti, ma anche il desiderio di una maggiore partecipazione politica e di un ritorno al welfare state, troppo spesso soffocato dalle politiche neoliberiste favorevoli ai privati e ai grandi gruppi finanziario-industriali. Dinamiche che, nonostante il benessere che hanno portato - e anche a causa di esso hanno esasperato una struttura sociale già in crisi appoggiando progetti di sviluppo spesso negativi per le masse popolari e la classe media che si sono viste escludere sempre più subdolamente dagli spazi urbani, sociali, economici e politici. 


\section{* L'autore}

Luca Zuccolo è dottorando (PhD student) in Storia Contemporanea presso il SUM (Istituto Italiano di Scienze Umane) di Napoli dove sta sviluppando una ricerca sulla stampa francofona ottomana e la sua rappresentazione dell'Impero d'Oriente. Già dottore magistrale in Storia d'Europa (Bologna, 2008), il suo campo di ricerca si rivolge allo sviluppo della modernità durante l'ultimo secolo dell'Impero Ottomano, al confronto/scontro tra modernità e tradizione in un contesto cosmopolita e allo sviluppo dei movimenti sociali che hanno preparato l'avvento della società turca contemporanea.

URL: < http://www.studistorici.com/2010/12/o1/luca-zuccolo/>

\section{Per citare questo articolo:}

ZUCCOLO, Luca, «Recensione: Ahdaf SOUEIF, Il Cairo, la mia città, la nostra rivoluzione, Roma, Donzelli Editore, 2013, 258 pp.», Diacronie. Studi di Storia Contemporanea : Le esposizioni: propaganda e costruzione identitaria, 29/6/2014,

URL: < http://www.studistorici.com/2014/6/29/zuccolo_numero_18/ >

\section{Diacronie Studi di Storia Contemporanea $\beta$ www.diacronie.it}

Risorsa digitale indipendente a carattere storiografico. Uscita trimestrale.

redazione.diacronie@hotmail.it

Comitato di redazione: Jacopo Bassi - Luca Bufarale - Elisa Grandi - Deborah Paci - Fausto Pietrancosta - Matteo Tomasoni - Luca Zuccolo 